\title{
Detection of Molecular Gas in Void Galaxies : Implications for Star Formation in Isolated Environments
}

\author{
M. Das \\ Indian Institute of Astrophysics, Bangalore, India \\ mousumi@iiap.res.in \\ T. Saito \\ Department of Astronomy, Graduate school of Science, The University of Tokyo, 7-3-1 \\ Hongo, Bunkyo-ku, Tokyo 133-0033, Japan \\ D. Iono \\ Chile Observatory, NAOJ, Japan \\ M. Honey \\ Indian Institute of Astrophysics, Bangalore, India \\ S. Ramya \\ Shanghai Astronomical Observatory, Shanghai, China
}

Received — 


\begin{abstract}
We present the detection of molecular gas from galaxies located in nearby voids using the $\mathrm{CO}(1-0)$ line emission as a tracer. The observations were done using the $45 \mathrm{~m}$ single dish radio telescope of the Nobeyama Radio Observatory (NRO). Void galaxies lie in the most underdense parts of our universe and a significant fraction of them are gas rich, late type spiral galaxies. Although isolated, they have ongoing star formation but appear to be slowly evolving compared to galaxies in denser environments. Not much is known about their star formation properties or cold gas content. In this study we searched for molecular gas in five void galaxies. The galaxies were selected based on their relatively high IRAS fluxes or $\mathrm{H} \alpha$ line luminosities, both of which signify ongoing star formation. All five galaxies appear to be isolated and two lie within the Bootes void. We detected $\mathrm{CO}(1-0)$ emission from four of the five galaxies in our sample and the molecular gas masses lie between $10^{8}-10^{9} M_{\odot}$. We did followup $\mathrm{H} \alpha$ imaging observations of three detected galaxies using the Himalayan Chandra Telescope and determined their star formation rates (SFR) from their H $\alpha$ fluxes. The SFR varies from $0.2-1 \mathrm{M}_{\odot} y r^{-1}$; which is similar to that observed in local galaxies. Our study indicates that although void galaxies reside in underdense regions, their disks contain molecular gas and have star formation rates similar to galaxies in denser environments. We discuss the implications of our results.
\end{abstract}

Subject headings: galaxies: evolution, galaxies : spiral, galaxies: star formation, galaxies: ISM, cosmology: large-scale structure of universe, radio lines: ISM 


\section{Introduction}

In the large scale structure of our universe, galaxies cluster along sheets, walls and filaments leaving large empty regions called voids in between (Kirshner et al. 1981; Geller \& Huchra 1989; Hoyle \& Vogeley 2004; Foster \& Nelson 2009; Sutter et al. 2012). They represent the most under-dense parts of our universe and can vary in size but typical values are 10 to $20 \mathrm{Mpc}$. For example the nearby Local Void is $\sim 23 \mathrm{Mpc}$ across (Tully et al. 2008) whereas the Bootes Void, which is the largest known void, is $60 \mathrm{Mpc}$ across (Kirshner et al. 1987). In the past two decades, large optical surveys have revealed that voids contain a small but significant population of galaxies (Grogin \& Geller 2000; Rojas et al. 2004; van de Weygaert et al. 2011). The smaller voids are mainly populated by small, gas rich, low surface brightness (LSB) dwarfs (Chengalur et al. 2015) and irregular galaxies (Karachentseva et al. 1999) but the larger voids also support a population of relatively bright galaxies that are late type, gas rich systems and often blue in color (Kreckel et al. 2011; Ricciardelli et al. 2014; Kreckel et al. 2015). This is in contradiction to cold dark matter (CDM) models of structure formation, where voids are predicted to be mainly populated by low luminosity galaxies (Peebles 2001). The presence of these blue void galaxies also indicates that there is ongoing star formation in voids. Even some early type elliptical (E) and lenticular (S0) void galaxies (that are mainly composed of old stars) are found to be relatively blue in color (Wegner \& Grogin 2008). However, although a signicant fraction of void galaxies do show star formation, they are in general evolving at a slower rate than galaxies in denser environments. This is evident from the color magnitude plot of these galaxies, in which the majority lie in the blue cloud region and not in the red region which is expected from an evolved galaxy population (Kreckel et al. 2012).

One of the main attractions of studying void galaxies is that they provide us an opportunity to study star formation in isolated environments. It is not clear what drives 
star formation activity in such underdense environments. In denser parts of the universe, galaxy interactions and mergers play an important role in triggering star formation in gas rich galaxies. The interactions perturb the axisymmetric structure of the galaxy disks leading to enhanced cloud collisions, star formation and gas infall to the nuclear regions. The enhanced nuclear gas surface densities can lead to nuclear star formation, AGN activity and outflows. This cycle of activity ultimately results in bulge growth and the overall evolution of galaxies from small star forming blue systems to the more evolved red galaxies. In voids, galaxy interactions (such as the galaxy pair CG 693-692) are rare. Some void galaxies have low luminosity companions such as LSB dwarfs and dwarf spheroidals that are not easy to spot in optical images but nevertheless perturb galaxy disks and trigger star formation. Such interactions are difficult to detect in optical surveys. One way of detecting them is through HI surveys (e.g. Szomoru et al. 1997) where they may appear to have disturbed HI morphologies. Although some void galaxies do show such features in their HI contours (e.g. BHI 1514+5155), interactions with visible or under-luminous companions are not high enough to explain the whole picture of star formation in voids and thus interactions may not be the main driver of star formation activity in these underdense environments (Grogin \& Geller 2000). Another possible driver of star formation activity in voids could be the slow accretion of cold gas by void galaxies from the inter-galactic medium (IGM) (Kreckel et al. 2012). Gas accretion can enhance the gas surface densities in disks. This can result in the formation of local disk instabilities which lead to disk star formation (Dekel \& Birnboim 2006; Kereš et al. 2005). Recent observations of HI gas filaments connecting galaxies in voids suggests that this process may be important for galaxy pairs or small groups (Beygu et al. 2013; Kreckel et al. 2012; Stanonik et al. 2009).

Void galaxies are also one of the only probes by which we can investigate the void substructure - does it exist and how is it traced by galaxies? In $\Lambda$ CDM theories of large scale structure (LSS) formation, voids evolve with time into larger, emptier volumes. In 
the process mass flows from voids into walls and filaments (Zel'dovich 1970; Icke 1984; Bond et al. 1996; Aragon-Calvo \& Szalay 2013). Isolated or small groups of galaxies are left behind within the voids, formed by the compression of filaments or walls as the voids merge (Sahni et al. 1994; Sheth \& van de Weygaert 2004; van de Weygaert et al. 2010). This void substructure, which in simulations appears as tenuous filaments and clusters of dark matter halos, can be traced by the distribution of void galaxies (Hahn et al. 2007; Aragón-Calvo et al. 2007; Cautun et al. 2014). Recent deep observations have shown that this void substructure exists (Alpaslan et al. 2014; Kreckel et al. 2012). But only really deep surveys will reveal the inner structure of voids and how it connects the galaxies residing within the voids (Penny et al. 2015). As shown in simulations there will also be gas flowing along these filaments and accreting onto the void galaxies. This can lead to star formation in the galaxy disks.

As a first step towards studying star formation and galaxy evolution in voids, we present a search for molecular gas in nearby void galaxies using the Nobeyama Radio telescope (NRO) using the $\mathrm{CO}(1-0)$ emission line as a tracer. Single dish observations will give an estimate of the molecular gas masses and the emission line profiles can reveal properties of the gas distribution, such as whether the gas is centrally concentrated or more extended in a rotating disk. There are only two earlier studies that have detected $\mathrm{CO}(1-0)$ in voids; we use those results to enhance our sample. We followed up our $\mathrm{CO}(1-0)$ detections with $\mathrm{H} \alpha$ observations of three detected galaxies and derived their star formation rates. In the following sections we describe our sample selection, the galaxy properties, observations and results. For all distances we have used $H_{0}=73 \mathrm{~km} \mathrm{~s}^{-1} \mathrm{Mpc}^{-1}$ and $\Omega=0.27$ (Komatsu et al. 2009). 


\section{Sample Selection}

We selected an initial sample of 12 galaxies from earlier studies of void galaxies (Cruzen et al. 2002; Weistrop et al. 1995; Szomoru et al. 1996) and the Void Galaxy Survey (VGS) which used SDSS images to select a sample of sixty void galaxies and study their HI/radio properties using the WSRT telescope (Kreckel et al. 2012). Our shortlisted galaxies had the following properties:

(i) Significant HI gas masses and stellar masses greater than $10^{9} M_{\odot}$. This is to ensure avoiding dwarf galaxies in our sample since previous studies of low surface brightness (LSB) dwarfs indicate that they are unlikely to have molecular gas (Das et al. 2006).

(ii) Signatures of ongoing star formation. We checked the SDSS spectra of the sample galaxies to confirm the presence of emission lines that are typical of star formation (e.g. $\mathrm{H} \alpha, \mathrm{H} \beta$ and $[\mathrm{OIII}])$.

(iii) For galaxies with IRAS fluxes, we selected only those that had $S_{100}>1.0 \mathrm{Jy}$.

(iv) None of the galaxies had been previously studied in $\mathrm{CO}(1-0)$ emission.

However, due to unfavourable weather conditions we were able to observe only five galaxies from this sample. They are listed in Table 1 and are briefly described below.

SBS1325+597 (IRASF 13254+5945, VGS 34) : This is a gas rich galaxy with a size of $D_{25} \sim 11.7 \mathrm{kpc}$ (Table 1) that has been studied as part of the Void Galaxy Survey (here after VGS) (Kreckel et al. 2011). The SDSS image of the galaxy shows a compact, red nucleus and a single, faint spiral arm. The SDSS optical spectrum has few emission lines suggesting only moderate star formation and shows no signatures of AGN activity. The HI is extended well beyond the optical radius and the outer isophotes are disturbed, which suggests that the galaxy maybe interacting with a distant companion galaxy.

SDSS 143052.33+551440.0 (SDSS1430+5514, VGS 44) : This is also a moderate size disk galaxy with a $r$ band disk radius of $\sim 3.6 k p c$ and an HI radius $<7 k p c$ (Kreckel et al. 
2012). There is no strong bulge or extended disk but the SDSS image reveals a blue nucleus that shows strong $\mathrm{H} \alpha$ emission in the optical spectrum, indicative of nuclear star formation. SDSS 153821.22+331105.1 (SDSS1538+3311, VGS 57) : This is the only galaxy in our sample that appears to have a bar. It has an extended disk of radius $17.9^{\prime \prime}$ or $\sim 8.1 k p c$ in the SDSS $r$ band image and an HI radius $<9 k p c$ (Kreckel et al. 2012). Though the galaxy is relatively blue and the SDSS nuclear spectrum shows strong $\mathrm{H} \alpha$ emission, other signatures of strong star formation such as [OI] emission which is associated with shocks, are lacking. The ongoing star formation is located mainly along the bar.

CG 598 (IRAS F14575+4228) : This is a distant but large galaxy in the Bootes void that has disk radius of $22.4^{\prime \prime}$ or $\sim 26.2 \mathrm{kpc}$ in the $k_{s}$ band and shows signs of strong star formation in its optical spectrum (e.g. $\mathrm{H} \alpha, \mathrm{H} \beta$ and [OII] emission) (Cruzen et al. 2002). It is gas rich and the HI gas disk is extended well beyond the optical disk Szomoru et al. (1996). In the SDSS $g$ band image, the galaxy appears to be accreting a smaller galaxy. The interaction probably triggered the burst of star formation.

SBS 1428+529 (IRAS F14288+5255) : This is also a distant galaxy located in the Bootes void. Like CG 598 it is large in size (radius $\sim 27^{\prime \prime}$ or $\sim 24.6 \mathrm{kpc}$ ) but appears to be isolated. It has a bright bulge, distinct spiral arms and a strong bar. It is the only galaxy in our sample that shows AGN activity; it hosts a Seyfert 2 type nucleus (Cruzen et al. 2002).

\section{CO Observations and Data Reduction}

The ${ }^{12} C O(J=1-0)$ single dish, emission line observations were done using the 45 m Nobeyama Radio Telescope during the period 14 - 25 April, 2013. At the CO rest frequency of $115.271204 \mathrm{GHz}$, the half-power beam width (HPBW) is $15^{\prime \prime}$ and the main beam efficieny is $30 \%$. We used the one beam (TZ1), dual polarization, sideband separating receiver (TZ). The signal was digitized to 3 bits before being transferred to the digital 
FX-type spectrometer SAM45, that has a bandwidth of $4 \mathrm{GHz}$ (Nakajima et al. 2008). Typical system temperatures were 160 - $260 \mathrm{~K}$. The chopper-wheel method was used for temperature calibration with a swtiching cycle of $10 \mathrm{~s}$. The resultant output had 4096 channels and a frequency resolution of $488 \mathrm{kHz}$.

The on source time for the first four galaxies varied between 1 to 1.5 hours. Due to poor weather conditions the fifth galaxy SBS 1428+529 was observed for only 25 minutes (Table 2). The pointing accuracy was about $\sim 2^{\prime \prime}$ to $\sim 4^{\prime \prime}$. The data was analysed using the NRO calibration tool NEWSTAR. Data with a wind velocity greater than $5 \mathrm{kms}^{-1}$ and data points with winding baselines were flagged out. The antenna temperature $\left(T_{A}\right)$ was converted to the main beam temperature $\left(T_{m b}\right)$ using a main beam efficiency of $\eta_{b}=0.3$ where $T_{A}=T_{m b} / \eta_{b}$. The spectra were converted from Kelvin to Jansky using the conversion factor of $2.4 \mathrm{Jy} \mathrm{K}^{-1}$.

\section{4. $\mathrm{H} \alpha$ Optical Observations and Data Reduction}

We conducted $\mathrm{H} \alpha$ observations of three galaxies in our sample, SBS $1325+597$, SDSS 143052.33+551440.0 and SDSS J153821.22+331105.1. The remaining two galaxies were not observed because we did not have suitable filters. The $\mathrm{H} \alpha$ observations were done using the Himalayan Faint Object Spectrograph Camera (HFOSC) which is mounted on the 2m Himalayan Chandra Telescope (HCT) installed at the Indian Astronomical Observatory (IAO), Hanle, India. HFOSC has a $2 \mathrm{~K} \times 4 \mathrm{~K}$ SITe CCD chip with a plate scale of 0.296 $\operatorname{arcsec}$ pixel $^{-1}$. The central $2 \mathrm{~K} \times 2 \mathrm{~K}$ covers a field of view of $10 \times 10 \operatorname{arcmin}^{2}$. The observations were obtained on 2014 April $11 \& 25$. For the galaxies SBS $1325+597$ and SDSS $143052.33+551440.0$ we used the $\mathrm{H} \alpha$ broad filter of band width $\sim 500 \AA$ to obtain the $\mathrm{H} \alpha$ images. But SDSS J153821.22+331105.1 is at a higher redshift of $z=0.023$ and the $\mathrm{H} \alpha$ line is shifted to $6714 \stackrel{\AA}{A}$. Hence for this galaxy we used the narrow-band [SII] filter of 
band width $\sim 100 \AA$ centered around the wavelength $6724 \AA$.

To obtain the continuum subtracted $\mathrm{H} \alpha$ images we followed the procedure as outlined below. The galaxy images were obtained in both the broad-band $r$ filter and narrow-band filters centered around the $\mathrm{H} \alpha$ line. The bias frames and twilight flats were used for preprocessing of the images. The data reduction was done using the standard packages

available in IRAF1. The frames were bias subtracted and flat field corrected using the master bias and master flat frames. The cosmic ray hits were removed using the task COSMICRAYS in IRAF. The images of the two different filters were aligned geometrically using the tasks GEOMAP \& GEOTRAN. The Point Spread Function (PSF) of both the broad and narrow-band frames were matched. The scale factor between the broad-band $r$ frame and the $\mathrm{H} \alpha$ frame was determined using the field stars. The continuum subtracted $\mathrm{H} \alpha$ images were obtained by subtracting the PSF matched scaled continuum $r$ band images from the narrow-band images as described in (Waller 1990). Flux calibration was done using the spectrophotometric standard star HZ44 (Oke 1990). Flux calibrated continuum subtracted $\mathrm{H} \alpha$ images are shown in Figures 3, 4 and 5.

\section{Results}

In this section we discuss our molecular gas detections (Table 1), followup H $\alpha$ observations for three of the detected galaxies (Table 3) and the implications of our results.

\footnotetext{
${ }^{1}$ Image Reduction \& Analysis Facility Software distributed by National Optical Astronomy Observatories, which are operated by the Association of Universities for Research in Astronomy, Inc., under co-operative agreement with the National Science Foundation
} 

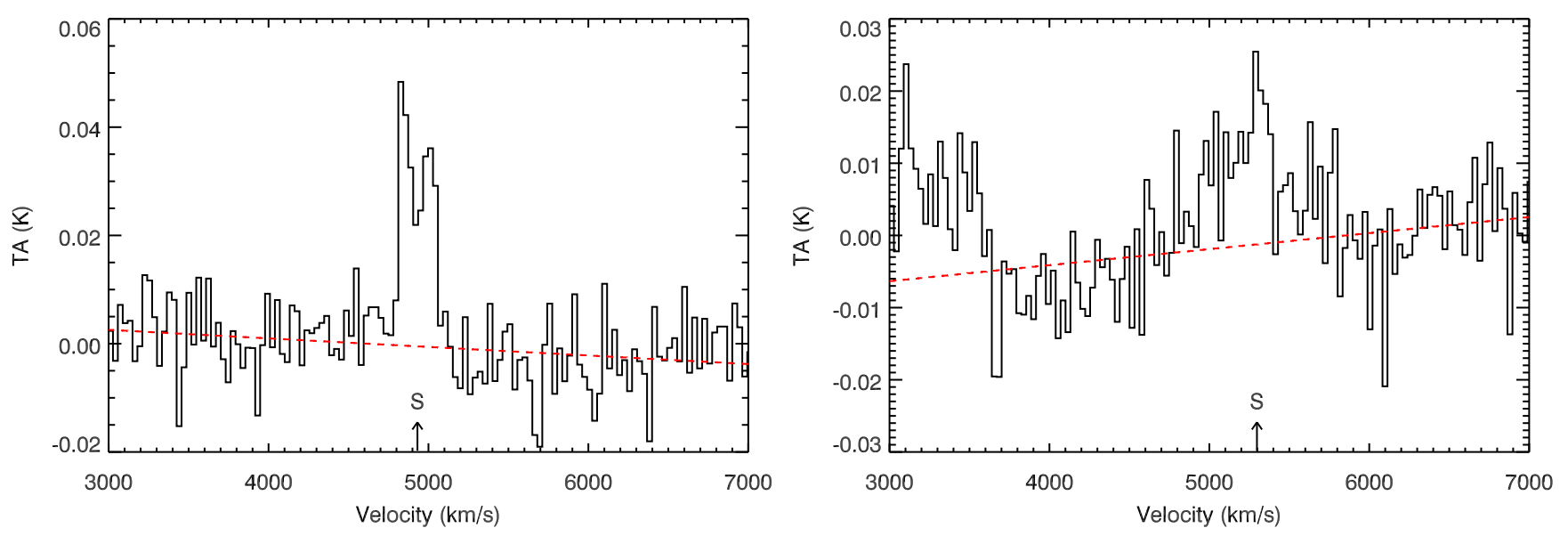

Fig. 1.- (a) The figure on the left shows the $\mathrm{CO}(1-0)$ spectrum observed from the galaxy SBS 1325+597. The emission line has a clear double horned profile. The dashed line in red represents the baseleine fitted to the spectrum. The systemic velocity of the galaxy, $v_{\text {sys }}=4917 \mathrm{kms}^{-1}$ (Kreckel at al. 2012) is marked with an 'S' and an arrow on the velocity axis. It lies in between the two emission peaks. This indicates that the molecular gas is in a rotating disk. (b) Figure on right shows the $\mathrm{CO}(1-0)$ spectrum from the galaxy SDSSJ 143052.33+551440.0. The fitted baseline is marked with a dashed red line and the systemic velocity of the galaxy, $v_{\text {sys }}=5295 \mathrm{kms}^{-1}$ (Kreckel at al. 2012) is marked. The emission can be clearly distinguished but is distributed over a range of velocities which suggests that the molecular gas distribution may not be centrally concentrated in the nucleus. 


\section{1. $\mathrm{CO}(1-0)$ detections :}

We have detected ${ }^{12} C O(J=1-0)$ emission from four of the five sample galaxies that we observed and the fluxes are listed in Table 2. The non-detection in SBS 1428+529 could be due to the short duration of the scan, which was limited by bad weather. The noise of the spectrum is $0.0024 \mathrm{~K}$. Assuming a typical $\mathrm{CO}(1-0)$ bandwidth of $250 \mathrm{~km} / \mathrm{s}$ we obtain $I_{C O}<0.6 \mathrm{~K} \mathrm{~km} \mathrm{~s}^{-1}$, which gives a limiting molecular gas mass of $0.59 \times 10^{9} M_{\odot}$ (Table 2). Of the four detections, SBS $1325+597$ has the most striking line profile; it has a double horned structure indicating a rotating disk of molecular gas (Figure 1a). The velocity separation of the peaks is $\sim 200 \mathrm{kms}^{-1}$. Assuming a disk inclination of 59.3 (Makarov et al. 2014) 2, the disk rotation is $116 \mathrm{~km} \mathrm{~s}^{-1}$. This is similar to the HI rotation speed in this galaxy derived from the HI observations of Kreckel et al. (2012). In SDSS 143052.33+551440.0 (Figures 1b) the CO line profile is fairly symmetric about the central systemic velocity of the galaxy but is distributed over a range of velocities which suggests that the molecular gas probably extends over the galaxy disk rather than concentrated within the nucleus. In both SDSS 153821.22+331105.1 and CG 598, the molecular gas is centrally peaked (Figure 1d and 2b). SDSS $153821.22+331105.1$ is barred and hence the molecular gas may have been driven into the center by the bar (Sakamoto et al. 1999).

\subsection{Molecular Gas Masses and surface densities :}

The CO fluxes in $\mathrm{K} \mathrm{km} \mathrm{s}^{-1}$ were converted to $\mathrm{Jy} \mathrm{km} / \mathrm{s}$ using a conversion factor $(\mathrm{Jy} / \mathrm{K})$ of 2.4. The CO line luminosity was determined using the relation $L_{C O}=3.25 \times 10^{7}\left(S_{C O} \Delta V / J_{y k m s}{ }^{-1}\right)\left(D_{L} / M p c\right)^{2}\left(\nu_{r e s}\right)^{-2}(1+z)^{-1}$ and the molecular gas

\footnotetext{
${ }^{2}$ http://leda.univ-lyon1.fr/
} 

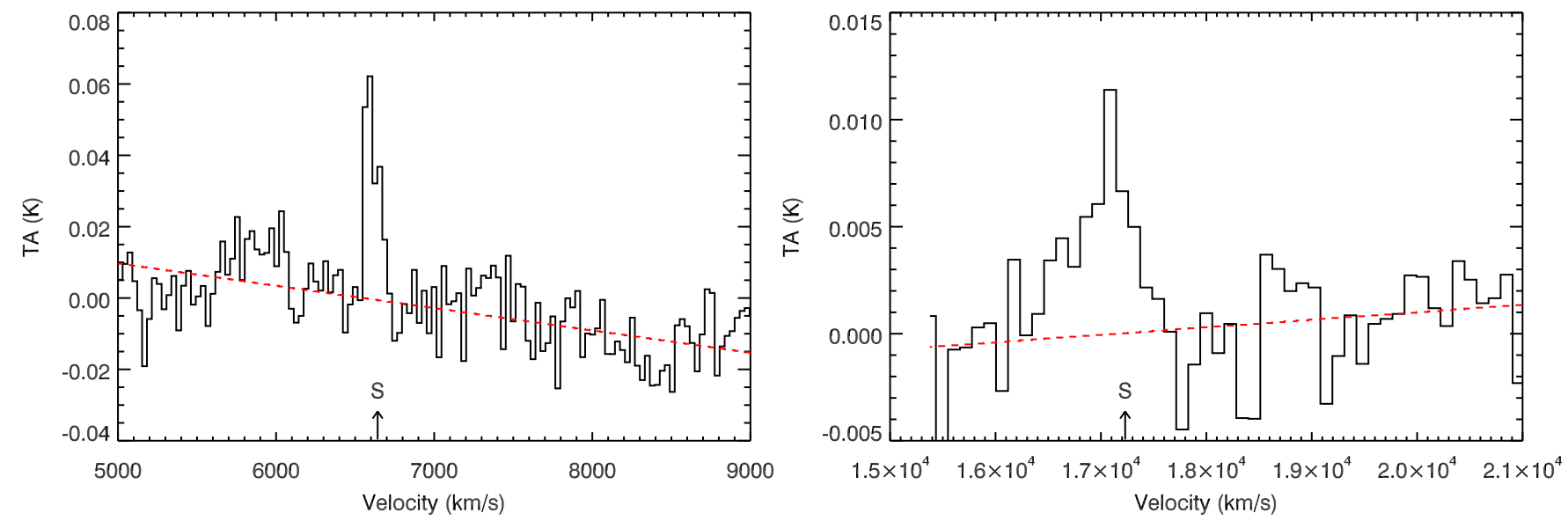

Fig. 2.- (a) The figure on the left shows the $\mathrm{CO}(1-0)$ spectrum of the galaxy SDSSJ 153821.22+331105.1. The emission is peaked about the systemic velocity of the galaxy, $v_{\text {sys }}=6630 \mathrm{kms}^{-1}$ (Kreckel at al. 2012) which suggests that the gas is concentrated in the center of the galaxy. The fitted baseline is marked with a dashed red line. (b) The figure on the right shows the $\mathrm{CO}(1-0)$ spectrum observed from $\mathrm{CG} 598$. The gas distribution is also symmetric about the center of the galaxy, which is marked at $v_{\text {sys }}=17226 \mathrm{kms}^{-1}$ (NED value) with and 'S'. 
masses were estimated using the relation $M\left(H_{2}\right)=\left[4.8 L_{C O}\left(K_{k m s^{-1}}\right)\right]$ (Solomon \& van den Bout 2005). The molecular gas masses lie in the range $(1-8) \times 10^{9} M_{\odot}$ (Table 2) which is comparable to the molecular gas masses observed in nearby bright galaxies that lie in denser environments (Helfer et al. 2003). Using the 2MASS galaxy sizes, we estimated the suface densities $\Sigma$ of the molecular gas distribution; it lies in the range $(3.8-30) M_{\odot} p c^{-2}$ (Table 3), which is also similar to that observed in nearby bright galaxies (Kennicutt 1998). Thus both the molecular gas masses and gas surface densitites are not unusually low, even though the galaxies are in low density environments.

\section{3. $\mathrm{H} \alpha$ Imaging and star formation rates :}

As mentioned earlier, due to lack of suitable filters we were able to do $\mathrm{H} \alpha$ imaging of only three galaxies in our sample, SBS $1325+597$, SDSS $143052.33+551440.0$ and SDSS J153821.22+331105.1 (Figures 3, 4, 5).

(i) In SBS $1325+597$ the $\mathrm{H} \alpha$ emisison is distributed over two regions on either side of the galaxy center, which indicates that it is only associated with the inner disk of the galaxy (Figure 3). This distribution agrees well with the $\mathrm{CO}(1-0)$ emission profile (Figure 1), which is double horned about the systemic velocity of the galaxy and indicates that the molecular gas is also distributed in a ring or torus about the galaxy center. In the SDSS optical image, it is clear that the galaxy has a red bulge but a bluish star forming disk. The $\mathrm{H} \alpha$ luminosity gives a moderate $\mathrm{SFR}$ of $0.2 \mathrm{M}_{\odot} \mathrm{yr}^{-1}$ (Table 3 ). The galaxy also shows extended UV emission in its GALEX image. The emission peak is offcenter from the nucleus and located east of the nucleus. Thus the overall picture of this galaxy is that of an isolated void galaxy with a moderately star forming disk that is rich in molecular gas. (ii) SDSS 143052.33+551440.0 is a small galaxy with a bright nucleus that appears blue in its SDSS optical image. The $\mathrm{H} \alpha$ emisison peaks close to the center of the galaxy but is 
extended over the enitire disk (Figure 4). The $\mathrm{H} \alpha$ luminosity gives a SFR of $0.6 \mathrm{M}_{\odot} \mathrm{yr}^{-1}$ (Table 3) which is relatively strong for a galaxy of this size. Both the $\mathrm{H} \alpha$ flux and the CO line emission appear to peak offcenter from the nucleus. The GALEX NUV emission is also extended over the galaxy. Thus SDSS 143052.33+551440.0 is a small, star forming, gas rich galaxy.

(iii) SDSS $153821.22+331105.1$ is the only barred galaxy in our sample. It appears to be an isolated, blue, star forming galaxy with a small bulge and faint spiral arms associated with the bar (Figure 5). The $\mathrm{H} \alpha$ emission peaks in the nucleus and is extended over the entire bar. This is similar to the molecular gas distribution which is sharply peaked about the systemic velocity of the galaxy (Figure 3) indicating that the molecular gas is concentrated in the center of the galaxy. It may have been driven into the nucleus by the bar. The bar may also have triggered the star formation. There is not much $\mathrm{H} \alpha$ emission detected from the disk but the total $\mathrm{H} \alpha$ luminosity gives a relatively high SFR of $1.02 \mathrm{M}_{\odot} \mathrm{yr}^{-1}$ (Table 3), probably due to the large $\mathrm{H} \alpha$ luminosity (i.e. SFR) along the bar. There is no GALEX image for this galaxy. Thus SDSS 153821.22+331105.1 appears to be a moderately sized barred galaxy that has strong star formation associated with the bar. It is a good example of ongoing secular evolution in a void galaxy, where the nuclear star formation contributes to bulge growth and the gas evolution is probably driven by the bar.

\subsection{Star formation efficiency and the critical mass surface density for star formation :}

The star formation efficiecy (SFE) is defined as the star formation rate divided by the ratio of the molecular gas mass to the dynamical or disk rotation timescales (Silk \& Mamon 2012). It is important as it indicates what fraction of the molecular gas is converted into

stars during star formation in a galaxy and the gas depletion timescales (Leroy et al. 2008). 
We estimated the approximate disk rotation timescales from the HI position velocity plots in Kreckel et al. (2012), using the flat rotation velocities $v$ and HI extent $r(H I)$. We have derived the SFE in three galaxies (Table 3). For the remaining two galaxies, disk rotation velocities were not available in the literature and hence we could not derive their SFE's. We used the relation $\mathrm{SFE}=\mathrm{SFR} / \mathrm{M}\left(\mathrm{H}_{2}\right) \times \frac{2 \pi r}{v}$ which becomes $\frac{S F R}{M\left(H_{2}\right)} \frac{D^{\prime \prime}}{v(k m / s)}\left(D_{M p c}\right)$ where $\mathrm{M}\left(\mathrm{H}_{2}\right)$ is the molecular gas mass in solar mass units, $v$ is the depojected rotation velocities (i.e. $\left.v_{o b s} / \operatorname{Sin} i\right), D^{\prime \prime}$ is the galaxy diameter in arcseconds and $D_{M p c}$ is the galaxy distance in Mpc. Of the three galaxies, SDSS $143052.33+551440.0$ has the highest SFE, probably because it is much smaller than the other two galaxies and yet has comparable SFRs. It is also very blue in its SDSS composite image, which suggests a high star formation rate. We have also calculated the critical surface density for star formation $\left(\Sigma_{\text {crit }}\right)$ (Kennicutt 1989), using again the approximate disk rotation velocities and total gas surface densities $\Sigma\left(H I+H_{2}\right.$, where $\Sigma_{\text {crit }}=\alpha \kappa \sigma_{\text {gas }} / 3.36 \mathrm{G}$. We assumed $\kappa=\sqrt{2} v / r, \alpha=0.69, \sigma_{\text {gas }}=10 \mathrm{~km} / \mathrm{s}$ where $v$ is the disk rotation velocity. For $\Sigma / \Sigma_{\text {crit }}>1$, the disk is unstable. Table 3 shows that all three star forming galaxies have gas surface densitites above the critical threshold. This is in contrast to LSB galaxies that have large HI masses but diffuse stellar disks that do not support star formation (Das et al. 2010).

\section{Comparison with previous $\mathrm{CO}$ detections} Beygu et al. 2013). In the earlier study by Sage et al., CO(1-0) was detected from four galaxies lying within the Bootes void. Of these four galaxies, two galaxies CG 910 and CG 684 show strong CO emission centered around their systemic velocities. We have adjusted the distances to $H_{o}=73 \mathrm{~km} \mathrm{~s}^{-1} \mathrm{Mpc}^{-1}$ and recalculated the molecular gas masses (Table 4). Both galaxies have molecular gas masses of the order of $10^{9}$ which is similar 
to that observed in our sample galaxies. CG 910 is an isolated galaxy with a relatively high disk inclination $\left(56.2^{\circ}\right)$ and a red bulge. It has a double horned $\mathrm{CO}(1-0)$ emission line profile with peaks separated by $\sim 370 \mathrm{~km} \mathrm{~s}^{-1}$, which indicates that the molecular gas disk is rotating with velocity $v_{r}=223 \mathrm{~km} \mathrm{~s}^{-1}$. The SDSS DR6 nuclear spectrum shows only weak $\mathrm{H} \alpha$ emisison which indicates that there is only moderate star formation in this galaxy. The other galaxy CG 684, is closely interacting with a companion galaxy (Szomoru et al. 1996). The blue color of its SDSS optical composite image suggests ongoing star formation (Cruzen et al. 2002), that has probably been triggered by a close tidal interaction with a companion galaxy. The $\mathrm{CO}(1-0)$ emission line has two broad peaks separated by $\sim 710 \mathrm{~km} \mathrm{~s}^{-1}$, which is too large to be due to disk rotation. Instead the two peaks probably represent gas disks in CG 684 and its companion galaxy. The strong $\mathrm{H} \alpha$ emission line in the SDSS (DR6) spectra of CG 684 indicates that there is ongoing nuclear star formation (Table 4).

The second study by Beygu et al. detected CO emission in the star forming galaxy Mrk 1477, which is denoted by VGS 31b in their void galaxy survey (Kreckel et al. 2012). The galaxy is part of a string of three galaxies that appear to lie along an HI filament in a void. Mrk 1477 is clearly tidally interacting with the nearest galaxy VGS 31a and has a bar as well as ring in its disk. Of the three galaxies only Mrk 1477 was studied in CO emission and the molecular gas mass is similar to our detections (Table 4). Unlike SBS1325+597 or CG 910, Mrk 1477 does not have a double horned CO(1-0) profile. Instead the molecular gas appears to be piled in the center of the disk. It has probably been driven into the galaxy center by the bar which shows strong streaming motion in $\mathrm{H} \alpha$ (Beygu et al. 2013). The nuclear SFR is significant and has a value of $0.45 M_{\odot} y r^{-1}$ (Table 4 ); the nucleus appears blue in the optical SDSS image of the galaxy.

Thus, to date, including our detections there are seven galaxies detected in CO 
emission. Their molecular gas masses lie in range $10^{8}-10^{9} M_{\odot}$, for g band luminosities 14.8 to 19 and SFRs 0.2 to 1.85. Also, of the seven galaxies detected in CO emission, five have blue bulges or inner disks indicating ongoing nuclear star formation. The two exceptions are CG 910 and SBS 1325+597, both of which have red bulges in their (composite) SDSS

images. However, it is interesting to note that both CG 910 and SBS 1325+597 have double horned $\mathrm{CO}(1-0)$ emission profiles, which shows that their molecular gas is distributed mainly in their disks and probably associated with disk star formation rather than nuclear star formation. The ongoing disk star formation in SBS 1325+597 is indicated by the faint blue color and the $\mathrm{H} \alpha$ emission from its disk (Figure 3). A drawback of our work is that our sample is small, even when previous detections of molecular gas are included (Table 4) and biased towards blue galaxies. Hence, it is not representative of the entire range of void galaxies and there is also no control sample. However, when we take into account the significant amount of star formation observed in void galaxies (e.g. Kreckel et al. 2012), it is probably safe to state that molecular gas is not rare in voids.

\section{Discussion}

Since molecular gas requires dust to form, its presence also indicates that there have been several cycles of star formation in these galaxies. In this section we address the following questions : (a) what drives star formation in these underdense environments and (b) are void galaxies dark matter dominated systems?

(i) Galaxies and the void substructure : Void galaxies form from the rare, small-scale peaks in the primordial density field and a larger volume will likely include more of these peaks. However, void galaxies may also form within the void substructure. In the hierarchical picture of void evolution, smaller voids merge to form larger voids (Sheth \& van de Weygaert 2004) causing mass to flow from the void centers to the 

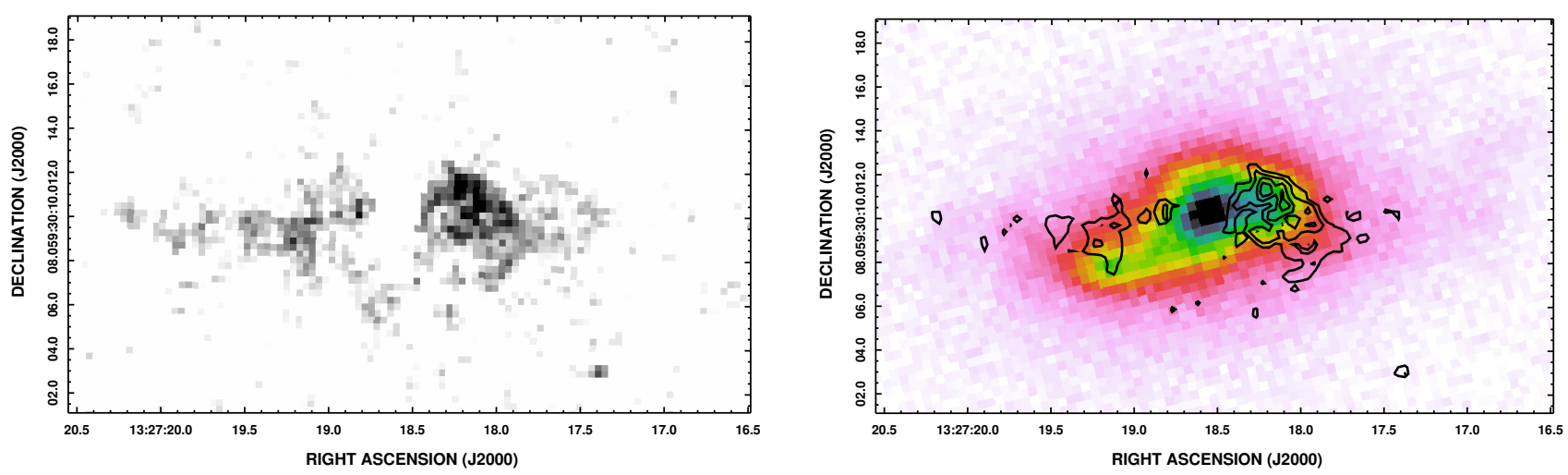

Fig. 3.- (a) The sky subtracted $\mathrm{H} \alpha$ image of SBS $1325+597$. The $\mathrm{H} \alpha$ emission is diffuse and distributed on either side of the nucleus. There appears to be more star formation west of the nucleus. (b) Contours of the $\mathrm{H} \alpha$ emission superimposed on the SDSS g band image of the galaxy. The contour levels are $0,0.12,0.18,0.24$ and 0.30 ; where the units are in counts $\mathrm{s}^{-1}$ and the sky subtracted background has a noise level of approximately 0.03 . The emission has a ringlike morphology and is associated with the disk. 

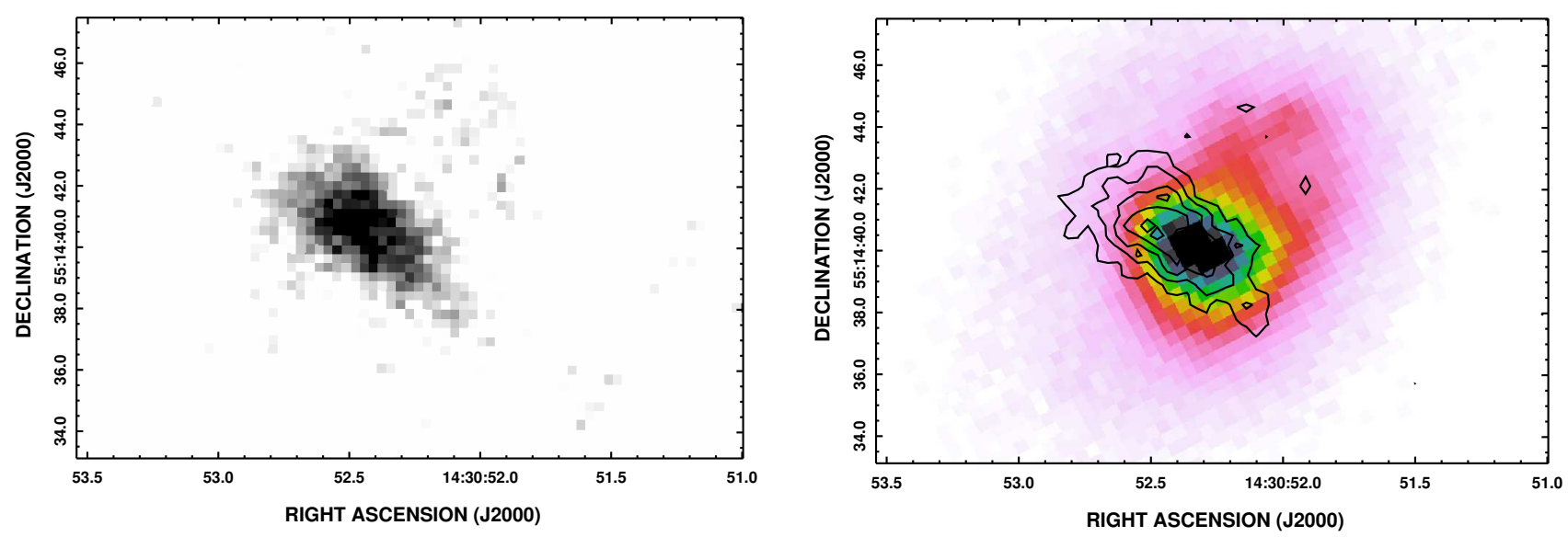

Fig. 4.- (a) The sky subtracted H $\alpha$ image of SDSS 143052.33+551440.0. The H $\alpha$ emission is distributed over the galaxy and extencec on one side. There is also diffuse emission lying to the northwest of the galaxy center that matches diffuse emission in the g band image as well. (b) Contours of $\mathrm{H} \alpha$ emission superimposed on the SDSS g band image of the galaxy. The contour levels are $0.27,0.45,0.63,0.81$ and 0.99 ; where the units are in counts $\mathrm{s}^{-1}$ and the sky subtracted background has a noise level of approximately 0.09. The $\mathrm{H} \alpha$ contours are extended on one side of the nucleus. 

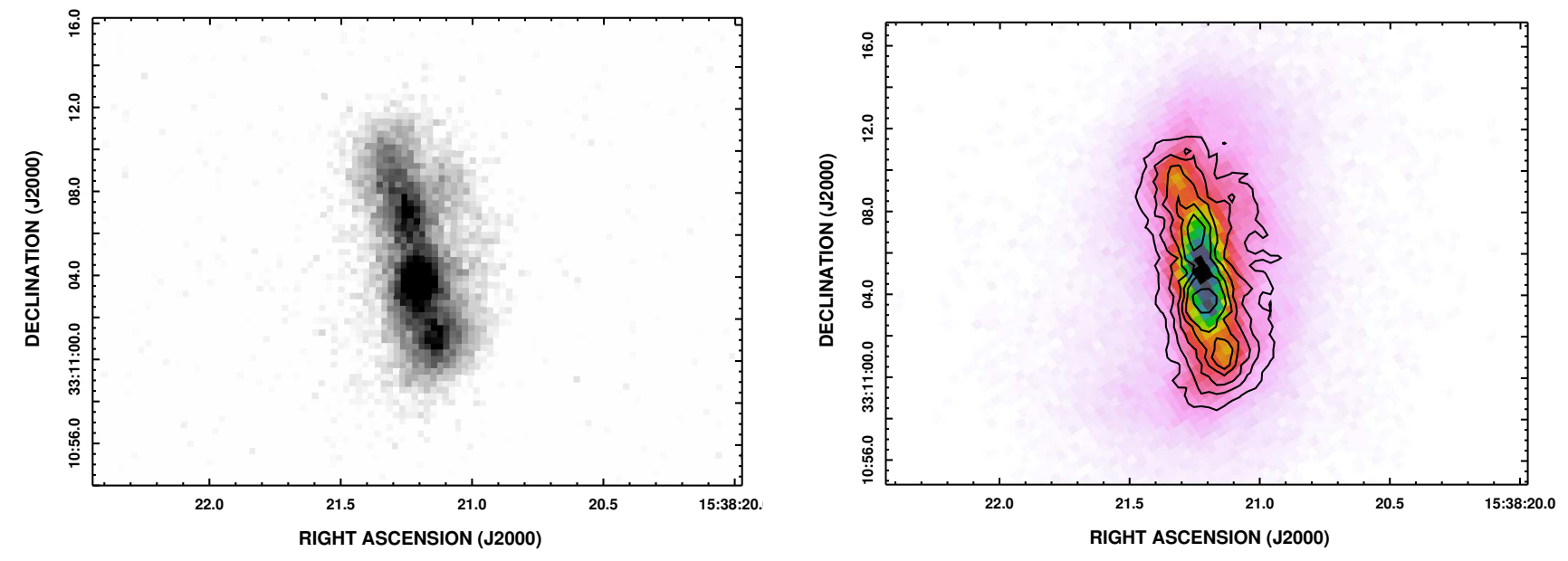

Fig. 5.- (a) The H $\alpha$ image of SDSS 153821.22+331105.1. The emission is strong and distributed along the bar in the galaxy. The flux density is highest in the nucleus and distributed over two regions along the bar. (b) Contours of $\mathrm{H} \alpha$ emission superimposed on the SDSS g band image of the galaxy. The contour levels are 0.27, 0.45, 0.63, 0.81 and 0.99; where the units are in counts $\mathrm{s}^{-1}$ and the sky subtracted background has a noise level of approximately 0.02. There is clearly strong star formation along the bar. The emission peaks on either side of the nucleus. 
surounding walls (Hahn et al. 2007; Aragón-Calvo et al. 2007; Aragon-Calvo \& Szalav 2013). In the process some matter may be left behind within the voids as filaments and mini-walls tracing a void substructure (Dubinski et al. 1993; Sahni et al. 1994). In this scenerio, small groups of galaxies can form within voids at places where the filaments or sheets intersect each other. So even though the large scale environemnt in voids is sparse, galaxy pairs (e.g. CG 692-CG 693) or small groups (e.g. VGS 31a,b,c) can form within the void substructure. There is now evidence that such filamentary structures within voids exist (Alpaslan et al. 2014; Beygu et al. 2013; Popescu et al. 1996). These galaxies interact or merge giving rise to star formation. Thus, some of the star formation that we observe in voids could be driven by the expansion and merging of the voids themselves. Thus, it is possible that we may observe more star forming galaxies and interacting systems in the larger voids, such as the Bootes void (Szomoru et al. 1996) where many more voids could have merged to form a larger void.

(ii) Slow gas accretion onto void galaxies from the IGM : Slow, cold gas accretion by galaxies along filaments has been shown to be important for galaxies with relatively low mass halos (Kereš et al. 2005; Dekel \& Birnboim 2006; Bouché et al. 2010). In void galaxies, cold gas from the intergalactic medium (IGM) may be accreted along the filaments that form part of the void substructure. The gas accretion increases the gas surface density as well as cools the disks. This can result in the formation of local disk instabilities which can trigger star formation. Cooler disks are also more prone to bar instabilities, which can again trigger star formation (e.g. SDSS 153821.22+331105.1) (Sellwood \& Wilkinson 1993). (iii) Are voids dominated by LSB galaxies? : $\Lambda$ CDM models of structure formation predict that voids are populated mainly by dark matter dominated galaxies, similar to the low surface brightness (LSB) galaxies that are observed at low redshifts (Peebles \& Nusser 2010). Although the smaller voids such as the Local Void, have a significant population of LSB dwarfs, the number density is not as high as predicted. Instead, surprisingly voids 
contain a significant population of blue galaxies (Peebles 2001). The main characterstics of LSB galaxies are their extended HI gas disks and low to moderate star formation rates. Molecular gas is very rare in these galaxies (Das et al. 2006) and their dust content is low (Rahman et al. 2007). Our study clearly shows that molecular gas is fairly abundant in void galaxies and star formation is also present. It suggests that voids are probably not dominated by LSB galaxies.

(iv) The dark matter content of SBS1325+597 (VGS 34) : This galaxy is the only one in our sample that has a HI position velocity (PV) plot that is extended enough to determine the flat rotation velocity in the galaxy disk. The two horned CO emission profile (Figure 1a) and the PV plot in Kreckel et al. (2012) indicate a projected flat disk velocity of $100 \mathrm{~km} / \mathrm{s}$, which when deprojected with an inclination angle of $59.3^{\circ}$ gives a disk rotation velocity $v_{c}=116.3 \mathrm{~km} / \mathrm{s}$. We used this velocity to obtain an approximate estimate of the dynamical mass. The PV plot contours extend out to an approximate radius of $30^{\prime \prime}$ which correponds to a lengthscale of $9.9 \mathrm{kpc}$, where $1^{\prime \prime}=330 \mathrm{pc}$. Thus the dynamical mass is $M_{d y n}=3.01 \times 10^{10} M_{\odot}$. To derive the stellar mass we used the $(\mathrm{M} / \mathrm{L})_{k}$ ratios derived from closed box models of chemical evolution (Bell \& de Jong 2001), where $\log (\mathrm{M} / \mathrm{L})=\mathrm{a}_{k}$ $+\mathrm{b}_{k}(\mathrm{~B}-\mathrm{V})$. Using the (g-r) magnitudes from SDSS DR12 and a conversion formula of $(B-V)=0.98($ g-r $)+0.22$ (Jester et al. 2005), we obtained a value $(M / L)_{k}=1.07$. Using the 2MASS total flux for this galaxy, we obtained a stellar mass of $M_{*}=1.27 \times 10^{10} M_{\odot}$ for SBS 1325+597. As listed in Table $2, \mathrm{M}\left(\mathrm{HI}+\mathrm{H}_{2}\right)=3.84 \times 10^{9} M_{\odot}$. Hence the baryonic mass is 0.55 of the total dynamical mass and so the galaxy is not dark matter dominated. Although this is one isolated case, the result may apply to other star forming galaxies in voids. Similar studies of a larger and more varied sample of void galaxies are necessary to determine the dark matter content of these galaxies. 


\section{Conclusions}

(i) We searched for molecular gas in a sample of five void galaxies using the $\mathrm{CO}(1-0)$ emission line. We detected molecular gas in four of the five observed galaxies. The molecular gas masses lie between $10^{8}-10^{9} M_{\odot}$.

(ii) We did follow-up $\mathrm{H} \alpha$ imaging observations of three of the detected galaxies and determined their star formation rates (SFRs) from their $\mathrm{H} \alpha$ fluxes. The SFR varies from $0.2-1 \mathrm{M}_{\odot} y r^{-1}$; which is similar to that observed in nearby star forming galaxies.

(iii) Our study and two others in the literature, indicate that although void galaxies reside in underdense regions, their disks contain molecular gas and may have star formation properties similar to galaxies in denser environments.

(vi) We derived the baryonic and dark matter content of one of our sample galaxies, SBS $1325+597$. We find that its baryonic content is 0.55 of the total dynamical mass and is hence not dark matter dominated. The result may apply to other star forming galaxies in voids.

M. Das would like to thank the anonymous referee for very useful comments that improved the paper. T. Saito is financially supported by a Research Fellowship from the Japan Society for the Promotion of Science for Young Scientists. S. Ramya kindly acknowledges the award of NSFC (Grant No. 11450110401) and President's International Fellowship Initiative (PIFI) awarded by the Chinese Academy of Sciences. This work was based on observations at the Nobeyama Radio Observatory (NRO). NRO is a branch of the National Astronomical Observatory of Japan, National Institutes of Natural Sciences. The optical observations were done at the Indian Optical Observatory (IAO) at Hanle. We thank the staff of IAO, Hanle and CREST, Hosakote, that made these obervations possible. The facilities at IAO and CREST are operated by the Indian Institute of Astrophysics, Bangalore. This research has made use of the NASA/IPAC Extragalactic Database (NED), 
which is operated by the Jet Propulsion Laboratory, California Institute of Technology, under contract with the National Aeronautics and Space Administration.

Our work has also used SDSS-III data. Funding for SDSS-III has been provided by the Alfred P. Sloan Foundation, the Participating Institutions, the National Science Foundation, and the U.S. Department of Energy Office of Science. The SDSS-III web site is http://www.sdss3.org/. SDSS-III is managed by the Astrophysical Research Consortium for the Participating Institutions of the SDSS-III Collaboration including the University of Arizona, the Brazilian Participation Group, Brookhaven National Laboratory, Carnegie Mellon University, University of Florida, the French Participation Group, the German Participation Group, Harvard University, the Instituto de Astrofisica de Canarias, the Michigan State/Notre Dame/JINA Participation Group, Johns Hopkins University, Lawrence Berkeley National Laboratory, Max Planck Institute for Astrophysics, Max Planck Institute for Extraterrestrial Physics, New Mexico State University, New York University, Ohio State University, Pennsylvania State University, University of Portsmouth, Princeton University, the Spanish Participation Group, University of Tokyo, University of Utah, Vanderbilt University, University of Virginia, University of Washington, and Yale University.

Facilities: Nobeyama Radio Observatory, Himalayan Chandra Telescope, SDSS 


\section{REFERENCES}

Alpaslan, M., Robotham, A. S. G., Obreschkow, D., et al. 2014, MNRAS, 440, L106

Aragón-Calvo, M. A., Jones, B. J. T., van de Weygaert, R., \& van der Hulst, J. M. 2007, A\&A, 474, 315

Aragon-Calvo, M. A., \& Szalay, A. S. 2013, MNRAS, 428, 3409

Bell, E. F., \& de Jong, R. S. 2001, ApJ, 550, 212

Beygu, B., Kreckel, K., van de Weygaert, R., van der Hulst, J. M., \& van Gorkom, J. H. 2013, AJ, 145, 120

Bond, J. R., Kofman, L., \& Pogosyan, D. 1996, Nature, 380, 603

Bouché, N., Dekel, A., Genzel, R., et al. 2010, ApJ, 718, 1001

Cautun, M., van de Weygaert, R., Jones, B. J. T., \& Frenk, C. S. 2014, MNRAS, 441, 2923

Chengalur, J. N., Pustilnik, S. A., Makarov, D. I., et al. 2015, MNRAS, 448, 1634

Cruzen, S., Wehr, T., Weistrop, D., Angione, R. J., \& Hoopes, C. 2002, AJ, 123, 142

Das, M., Boone, F., \& Viallefond, F. 2010, A\&A, 523, A63

Das, M., O’Neil, K., Vogel, S. N., \& McGaugh, S. 2006, ApJ, 651, 853

Dekel, A., \& Birnboim, Y. 2006, MNRAS, 368, 2

Dubinski, J., da Costa, L. N., Goldwirth, D. S., Lecar, M., \& Piran, T. 1993, ApJ, 410, 458

Foster, C., \& Nelson, L. A. 2009, ApJ, 699, 1252

Geller, M. J., \& Huchra, J. P. 1989, Science, 246, 897 
Grogin, N. A., \& Geller, M. J. 2000, AJ, 119, 32

Hahn, O., Porciani, C., Carollo, C. M., \& Dekel, A. 2007, MNRAS, 375, 489

Helfer, T. T., Thornley, M. D., Regan, M. W., et al. 2003, ApJS, 145, 259

Hoyle, F., \& Vogeley, M. S. 2004, ApJ, 607, 751

Icke, V. 1984, MNRAS, 206, 1P

Jester, S., Schneider, D. P., Richards, G. T., et al. 2005, AJ, 130, 873

Karachentseva, V. E., Karachentsev, I. D., \& Richter, G. M. 1999, A\&AS, 135, 221

Kennicutt, Jr., R. C. 1989, ApJ, 344, 685

—. 1998, ApJ, 498, 541

Kereš, D., Katz, N., Weinberg, D. H., \& Davé, R. 2005, MNRAS, 363, 2

Kirshner, R. P., Oemler, Jr., A., Schechter, P. L., \& Shectman, S. A. 1981, ApJ, 248, L57

—. 1987, ApJ, 314, 493

Komatsu, E., Dunkley, J., Nolta, M. R., et al. 2009, ApJS, 180, 330

Kreckel, K., Croxall, K., Groves, B., van de Weygaert, R., \& Pogge, R. W. 2015, ApJ, 798, L15

Kreckel, K., Platen, E., Aragón-Calvo, M. A., et al. 2012, AJ, 144, 16

-. 2011, AJ, 141, 4

Leroy, A. K., Walter, F., Brinks, E., et al. 2008, AJ, 136, 2782

Makarov, D., Prugniel, P., Terekhova, N., Courtois, H., \& Vauglin, I. 2014, A\&A, 570, A13 
Nakajima, T., Sakai, T., Asayama, S., et al. 2008, PASJ, 60, 435

Oke, J. B. 1990, AJ, 99, 1621

Peebles, P. J. E. 2001, ApJ, 557, 495

Peebles, P. J. E., \& Nusser, A. 2010, Nature, 465, 565

Peimbert, M., \& Torres-Peimbert, S. 1992, A\&A, 253, 349

Penny, S. J., Brown, M. J. I., Pimbblet, K. A., et al. 2015, ArXiv e-prints, arXiv:1508.06186

Popescu, C. C., Hopp, U., Hagen, H. J., \& Elsaesser, H. 1996, A\&AS, 116, 43

Rahman, N., Howell, J. H., Helou, G., Mazzarella, J. M., \& Buckalew, B. 2007, ApJ, 663, 908

Ricciardelli, E., Cava, A., Varela, J., \& Quilis, V. 2014, MNRAS, 445, 4045

Rojas, R. R., Vogeley, M. S., Hoyle, F., \& Brinkmann, J. 2004, ApJ, 617, 50

Sage, L. J., Weistrop, D., Cruzen, S., \& Kompe, C. 1997, AJ, 114, 1753

Sahni, V., Sathyaprakah, B. S., \& Shandarin, S. F. 1994, ApJ, 431, 20

Sakamoto, K., Okumura, S. K., Ishizuki, S., \& Scoville, N. Z. 1999, ApJ, 525, 691

Sargsyan, L. A., \& Weedman, D. W. 2009, ApJ, 701, 1398

Sellwood, J. A., \& Wilkinson, A. 1993, Reports on Progress in Physics, 56, 173

Sheth, R. K., \& van de Weygaert, R. 2004, MNRAS, 350, 517

Silk, J., \& Mamon, G. A. 2012, Research in Astronomy and Astrophysics, 12, 917

Stanonik, K., Platen, E., Aragón-Calvo, M. A., et al. 2009, ApJ, 696, L6 
Sutter, P. M., Lavaux, G., Wandelt, B. D., \& Weinberg, D. H. 2012, ApJ, 761, 44

Szomoru, A., van Gorkom, J. H., \& Gregg, M. D. 1996, AJ, 111, 2141

Tully, R. B., Shaya, E. J., Karachentsev, I. D., et al. 2008, ApJ, 676, 184

van de Weygaert, R., Platen, E., Tigrak, E., et al. 2010, in Astronomical Society of the Pacific Conference Series, Vol. 421, Galaxies in Isolation: Exploring Nature Versus Nurture, ed. L. Verdes-Montenegro, A. Del Olmo, \& J. Sulentic, 99

van de Weygaert, R., Kreckel, K., Platen, E., et al. 2011, The Void Galaxy Survey, ed. I. Ferreras \& A. Pasquali, 17

Waller, W. H. 1990, PASP, 102, 1217

Wegner, G., \& Grogin, N. A. 2008, AJ, 136, 1

Weistrop, D., Hintzen, P., Liu, C., et al. 1995, AJ, 109, 981

Zel'dovich, Y. B. 1970, A\&A, 5, 84 
Table 1. Sample Galaxies observed in CO(1-0) emisison with NRO

\begin{tabular}{|c|c|c|c|c|c|c|c|c|}
\hline $\begin{array}{c}\text { galaxy } \\
\text { name }\end{array}$ & $\begin{array}{c}\text { RA } \\
(\mathrm{J} 2000)\end{array}$ & $\begin{array}{c}\text { DEC } \\
(\mathrm{J} 2000)\end{array}$ & $\begin{array}{c}D_{L} \\
(\mathrm{Mpc})\end{array}$ & $\begin{array}{c}\text { redshift } \\
(\mathrm{z})\end{array}$ & type & $\begin{array}{c}\text { diameter } \\
\left({ }^{\prime \prime}\right)\end{array}$ & $\begin{array}{c}\mathrm{g} \\
\text { magnitude }\end{array}$ & $\begin{array}{c}\text { void } \\
\text { name }\end{array}$ \\
\hline SBS $1325+597$ & $13 \mathrm{~h} 27 \mathrm{~m} 18.6 \mathrm{~s}$ & $+59 \mathrm{~d} 30 \mathrm{~m} 10 \mathrm{~s}$ & 70.4 & 0.016 & Sm, HII & $36.0\left(\mathrm{k}_{s}\right)$ & 16.0 & Ursa Minor I \\
\hline SDSS 143052 & $14 \mathrm{~h} 30 \mathrm{~m} 52.3 \mathrm{~s}$ & $+55 \mathrm{~d} 14 \mathrm{~m} 40 \mathrm{~s}$ & 76.6 & 0.018 & Extend. & 76.0 & $19.5^{d}$ & Ursa Minor I \\
\hline SDSS 153821 & $15 \mathrm{~h} 38 \mathrm{~m} 21.2 \mathrm{~s}$ & $+33 \mathrm{~d} 11 \mathrm{~m} 05 \mathrm{~s}$ & 97.6 & 0.022 & $\mathrm{Sd}$ & $17.88\left(\mathrm{r}^{d}\right)$ & 15.3 & $\ldots$. \\
\hline CG 598 & $14 \mathrm{~h} 59 \mathrm{~m} 20.6 \mathrm{~s}$ & $+42 \mathrm{~d} 16 \mathrm{~m} 10 \mathrm{~s}$ & 248.0 & 0.057 & HII, Sbrst & $44.7\left(\mathrm{k}_{s}\right)$ & 16.4 & Bootes \\
\hline SBS $1428+529$ & $14 \mathrm{~h} 30 \mathrm{~m} 31.2 \mathrm{~s}$ & $+52 \mathrm{~d} 42 \mathrm{~m} 26 \mathrm{~s}$ & 191.0 & 0.044 & $\mathrm{Sb}, \mathrm{Sy} 2$ & $53.9\left(\mathrm{k}_{s}\right)$ & 15.2 & Bootes \\
\hline
\end{tabular}

${ }^{a}$ SDSS 143052 full name is SDSS 143052.33+551440.0 1 and SDSS 153821 full name is SDSS 153821.22+331105.1.

${ }^{b}$ Void identifications for the first two galaxies was obtained from Kreckel et al. (2011).

${ }^{\mathrm{c}}$ Distances for all galaxies was obtained from NED, except for SDSS $143052.33+551440.0$ which was obtained from Kreckel et al. (2012).

${ }^{\mathrm{d}}$ The optical size of the galaxy SDSS $143052.33+551440.01$ was obtained from Kreckel et al. (2012) and for SDSS 153821.22+331105.1 from SDSS. 
Table 2. CO emisison and derived gas masses

\begin{tabular}{|c|c|c|c|c|c|c|c|}
\hline $\begin{array}{l}\text { galaxy } \\
\text { name }\end{array}$ & $\begin{array}{c}\mathrm{t}_{\text {obs }} \\
\text { (hours) }\end{array}$ & $\begin{array}{c}\mathrm{S}_{C O} \Delta v \\
\left(\mathrm{~K} \mathrm{~km} \mathrm{~s}^{-1}\right)\end{array}$ & $\begin{array}{c}\mathrm{L}_{C O}\left(10^{8}\right) \\
\left(\mathrm{K} \mathrm{km} \mathrm{s}^{-1} \mathrm{pc}^{2}\right)\end{array}$ & $\begin{array}{l}\mathrm{H}_{2} \text { Mass } \\
\left(\begin{array}{ll}10^{9} & M_{\odot}\end{array}\right)\end{array}$ & $\begin{array}{l}\text { Surface density } \\
\Sigma_{H_{2}} \mathrm{M}_{\odot} p c^{-2}\end{array}$ & $\begin{array}{l}\text { HI mass } \\
10^{9} M_{\odot}\end{array}$ & $\frac{M\left(H_{2}\right)}{M(H I)}$ \\
\hline SBS $1325+597$ & $1 \mathrm{~h} 12 \mathrm{~m}$ & $10.7 \pm 0.2$ & $(3.1 \pm 0.1)$ & $1.5 \pm 0.03$ & $12.4 \pm 0.3$ & $2.4 \pm 0.3$ & 0.6 \\
\hline SDSS 143052 & $1 \mathrm{~h} 16 \mathrm{~m}$ & $7.0 \pm 0.2$ & $(2.4 \pm 0.1)$ & $1.1 \pm 0.03$ & $27.5 \pm 0.7$ & $0.50 \pm 0.1$ & 2.3 \\
\hline SDSS 153821 & $1 \mathrm{~h} 31 \mathrm{~m}$ & $6.4 \pm 0.2$ & $(3.5 \pm 0.1)$ & $1.7 \pm 0.05$ & $29.9 \pm 0.9$ & $0.7 \pm 0.2$ & 2.5 \\
\hline CG 598 & $2 \mathrm{~h} 26 \mathrm{~m}$ & $5.2 \pm 0.1$ & $(18.0 \pm 0.3)$ & $8.5 \pm 0.1$ & $3.8 \pm 0.1$ & $5.3 \pm 0.8$ & 1.6 \\
\hline SBS $1428+529$ & Oh $25 \mathrm{~m}$ & $<0.6$ & $<1.23$ & $<0.6$ & $\ldots$ & $2.0 \pm 0.4$ & $<0.4$ \\
\hline
\end{tabular}

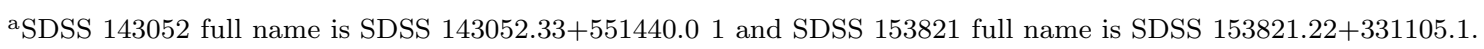

${ }^{\mathrm{b}}$ To derive the approximate molecular gas surface densities $\Sigma\left(H_{2}\right)$ we used the galaxy diameters and distances listed in Table 1.

${ }^{\mathrm{c}}$ The HI masses for the galaxies SBS $1325+597$ (VGS 34), SDSS $143052.33+551440.0$ (VGS 44) and SDSS 153821.22+331105.1 (VGS 57) were obtained from Kreckel et al. (2012) and adjusted for the distances listed in Table 1. For CG 598 and SBS 1428+529 we used data from Szomoru et al. (1996). 
Table 3. H $\alpha$ luminosities, star formation rates and star formation thresholds

\begin{tabular}{|c|c|c|c|c|c|c|c|}
\hline $\begin{array}{l}\text { galaxy } \\
\text { name }\end{array}$ & $\begin{array}{c}\text { H } \alpha \text { Flux } \\
10^{-14} \mathrm{erg} \mathrm{cm}^{-2} \mathrm{~s}^{-1}\end{array}$ & $\begin{array}{l}\mathrm{H} \alpha \text { luminosity } \\
10^{40} \mathrm{erg} \mathrm{s}^{-1}\end{array}$ & $\begin{array}{c}\mathrm{SFR} \\
\mathrm{M}_{\odot \mathrm{yr}^{-1}}\end{array}$ & $\mathrm{SFE}^{b}$ & $\begin{array}{c}\mathrm{M}\left(\mathrm{HI}+\mathrm{H}_{2}\right) \\
10^{9} M_{\odot}\end{array}$ & $\begin{array}{l}\text { Disk Rotation } \\
\qquad \mathrm{km} \mathrm{s}^{-1}\end{array}$ & ${\frac{\Sigma\left(H I+H_{2}\right)^{c}}{\Sigma_{c r i t}}}^{c}$ \\
\hline SBS $1325+597$ & 4.44 & 2.49 & 0.20 & 0.03 & 3.89 & 116.30 & 2.7 \\
\hline SDSS 143052 & 11.65 & 7.61 & 0.60 & 0.21 & 1.60 & 145.62 & 1.5 \\
\hline SDSS 153821 & 11.94 & 1.29 & 1.02 & 0.05 & 2.35 & 200.0 & 1.4 \\
\hline CG 598 & $\ldots$ & $\ldots$ & $1.48^{d}$ & $\ldots$ & 13.85 & $\ldots$ & $\ldots$ \\
\hline SBS $1428+529$ & $5.65^{e}$ & 23.3 & 1.85 & $\ldots$ & $<2.61$ & $\ldots$ & $\ldots$ \\
\hline
\end{tabular}

${ }^{a}$ SDSS 143052 full name is SDSS 143052.33+551440.0 1 and SDSS 153821 full name is SDSS 153821.22+331105.1.

${ }^{\mathrm{b}}$ The star formation efficiency (SFE) is defined as the SFR divided by the ratio of molecular gas mass to disk rotation timescales (see Section 5 for details).

${ }^{\mathrm{c}}$ This is the ratio of the total gas surface density and the critical surface density for star formation $\Sigma\left(H I+H_{2}\right) / \Sigma_{c r i t}>1$ in star forming galaxies (Kennicutt 1998). Its calculation is discussed in Section 5.

$\mathrm{d}$ The star formation rate (SFR) is derived from the UV flux (Sargsyan \& Weedman 2009).

eThe H $\alpha$ flux for SBS $1428+529$ is from Weistrop et al. (1995). 
Table 4. Comparison of molecular gas detection in other void galaxies

\begin{tabular}{ccccccc}
\hline \hline galaxy & Distance & $\mathrm{H}_{2}$ Mass & HI Mass & $\frac{M\left(H_{2}\right)}{M(H I)}$ & SFR & Reference \\
name & Mpc & $10^{9} M_{\odot}$ & $10^{9} M_{\odot}$ & & $\mathrm{M}_{\odot \mathrm{yr}^{-1}}$ & \\
\hline CG 910 & 188 & 4.2 & $\ldots$ & $\ldots$ & $\ldots$ & Sage et al. (1997) \\
CG 684 & 204 & 4.7 & 9.2 & 0.51 & $1.83^{a}$ & $\begin{array}{c}\text { Sage et al., Szomoru et al.(1997) } \\
\text { VGS 31b (Mrk 1477) }\end{array}$ \\
\hline
\end{tabular}

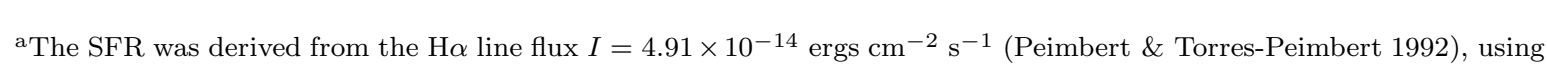
the relation relation $\mathrm{SFR}=\mathrm{L}(\mathrm{H} \alpha) / 1.26 \times 10^{42}$ (Kennicutt et al. 1998).

${ }^{\mathrm{b}}$ We have used the $L_{C O}{ }^{\prime}$ from Beygu et al. (2013) and the relation $\mathrm{M}\left(\mathrm{H}_{2}\right)=4.8 L_{C O}$ ' to determine this value.

${ }^{\mathrm{c}}$ The SFR was derived from the SDSS DR10 H $\alpha$ line flux of $I=11.56 \times 10^{-14} \mathrm{ergs} \mathrm{cm}^{-2} \mathrm{~s}^{-1}$. 